\title{
Small Deviations of Stable Processes via Metric Entropy
}

\author{
Wenbo V. Li ${ }^{* \dagger}$
}

Werner Linde $\ddagger$

Technical Report No. 2002-11

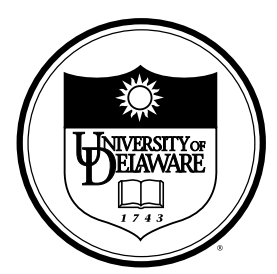

DEPARTMENT

$\mathrm{OF}$

MATHEMATICAL SCIENCES

\author{
University of Delaware \\ Newark, Delaware
}

\footnotetext{
*Supported in part by NSF Grant DMS-0204513

$\dagger$ Department of Mathematical Sciences, University of Delaware, Newark, DE 19711. Email: wli@math.udel.edu

${ }^{\ddagger}$ Friedrich-Schiller-Universität Jena, Institut für Stochastik, Ernst-Abbe-Platz 1-4, 07743 Jena, Germany. E-mail: lindew@minet.uni-jena.de
} 


\title{
Small Deviations of Stable Processes via Metric Entropy
}

\author{
Wenbo V. $\mathrm{Li}^{1,2} \quad$ Werner Linde ${ }^{3}$
}

\begin{abstract}
Let $X=(X(t))_{t \in T}$ be a symmetric $\alpha$-stable, $0<\alpha<2$, process with paths in the dual $E^{*}$ of a certain Banach space $E$. Then there exists a (bounded, linear) operator $u$ from $E$ into some $L_{\alpha}(S, \sigma)$ generating $X$ in a canonical way. The aim of this paper is to compare the degree of compactness of $u$ with the small deviation (ball) behavior of $\phi(\varepsilon)=-\log \mathbb{P}\left(\|X\|_{E^{*}}<\varepsilon\right)$ as $\varepsilon \rightarrow 0$. In particular, we prove that a lower bound for the metric entropy of $u$ implies a lower bound for $\phi(\varepsilon)$ under an additional assumption on $E$. As applications we obtain lower small deviation estimates for weighted $\alpha$-stable Levy motions, linear fractional $\alpha$-stable motions and $d$-dimensional $\alpha$-stable Levy sheets. Our results rest upon an integral representation of $L_{\alpha}$-valued operators as well as on small deviation results for Gaussian processes due to Kuelbs and Li and to the authors.
\end{abstract}

Abbreviated title: Small Deviations of Stable Processes

Keywords: Stable processes, small deviation, metric entropy.

AMS 2000 Subject Classifications: Primary: 60G52 ; Secondary: 47B06, 60G15, 47G10 .

\footnotetext{
${ }^{1}$ Supported in part by NSF Grant DMS-0204513

${ }^{2}$ Department of Mathematical Sciences, University of Delaware, Newark, DE 19711. E-mail: wli@math.udel.edu

${ }^{3}$ Friedrich-Schiller-Universität Jena, Institut für Stochastik, Ernst-Abbe-Platz 1-4, 07743 Jena, Germany. E-mail: lindew@minet.uni-jena.de
} 


\section{Introduction}

Let $T \neq \emptyset$ be an index set and let $E$ be a Banach space of functions over $T$. Assume that for some separable Hilbert space with orthogonal normal base (ONB) $\left(f_{k}\right)_{k \geq 1}$ and a given operator $v$ from $H$ into $E$ the sum $\sum_{k=1}^{\infty} \xi_{k} v\left(f_{k}\right)$ exists a.s. in $E$. Here and later on we always denote $\left(\xi_{k}\right)_{k \geq 1}$ as an i.i.d. sequence of standard normal distributed random variables, i.e. $\xi_{k} \sim \mathcal{N}(0,1)$. Then

$$
X(t):=\sum_{k=1}^{\infty} \xi_{k}\left(v f_{k}\right)(t), \quad t \in T,
$$

defines a centered Gaussian process $X$ over $T$ possessing a.s. paths in $E$. As discovered in Kuelbs and $\mathrm{Li}^{[5]}$ and completed in $\mathrm{Li}$ and Linde ${ }^{[6]}$, the degree of compactness of $v$ is tightly related to the behavior of the so-called small deviation (or small ball) function

$$
\phi(\varepsilon)=-\log \mathbb{P}\left(\|X\|_{E}<\varepsilon\right) \quad \text { as } \quad \varepsilon \rightarrow 0
$$

One very useful way to measure the degree of compactness of an operator is the behavior of its entropy numbers. They are defined as follows: Let $v$ map a Banach space $E$ into another Banach space $F$, then

$$
e_{n}(v):=\inf \left\{\varepsilon>0: v\left(B_{E}\right) \subseteq \bigcup_{j=1}^{2^{n-1}}\left\{v\left(x_{j}\right)+\varepsilon B_{F}\right\}, x_{j} \in B_{E}\right\}
$$

where $B_{E}$ and $B_{F}$ are the (closed) unit balls in $E$ or $F$, respectively.

The precise relation between the sequence $\left(e_{n}(v)\right)_{n \geq 1}$ and the behavior of (1.2) asserts the following.

Proposition 1.1 Let $X$ and $v$ be in relation (1.1) and let $\theta \in(0,2)$ be given. Defining $\lambda>0$ by $1 / \lambda=1 / \theta-1 / 2$, the following are equivalent:

$$
\begin{array}{r}
e_{n}(v) \approx n^{-1 / \theta} \quad \text { as } \quad n \rightarrow \infty \\
-\log \mathbb{P}\left(\|X\|_{E}<\varepsilon\right) \approx \varepsilon^{-\lambda} \quad \text { as } \quad \varepsilon \rightarrow 0 .
\end{array}
$$

Here and throughout the paper, $f(x) \approx g(x)$ as $x \rightarrow a$ means $c \leq f(x) / g(x) \leq C$ for $x$ near to $a$ and with some absolute constants $0<c \leq C<\infty$.

A natural question is whether or not there are stochastic processes other than Gaussian that allow an entropy description of their small deviation behavior similar to ones in Proposition 1.1. Natural candidates are symmetric $\alpha$-stable processes with $0<\alpha<2$. This seems very likely based on our investigation in this paper, yet several difficult problems appear 
even in the basic formulation. For example, in contrast to the Gaussian case, not every $\alpha$-stable process allows a representation (1.1) with standard $\alpha$-stable r.v.'s instead of the $\xi_{k}$ 's. Consequently, at the beginning it is not clear at all for which operator the degree of compactness should be investigated. Another important difference is that almost all stable processes of interest have non-continuous paths. Thus the Banach space $C(T)$ of continuous functions over $T$ is not suitable in this case. More suitable Banach spaces such as, e.g. $B(T)$, the space of bounded functions over $T$, are non-separable, hence measurability problems arise.

One possible way to overcome all these difficulties is to regard a stable process $X$ as random variable with values in a dual Banach space $E^{*}$ endowed with the weak-*-topology. This is the setup we use and under some natural assumptions on $X$ there exists an operator $u: E \rightarrow L_{\alpha}$ satisfying

$$
\mathbb{E} e^{i\langle x, X\rangle}=e^{-\|u(x)\|_{\alpha}^{\alpha}}, \quad x \in E .
$$

The main result of this paper (Theorem 4.5 below) asserts in particular the following onesided extension of Proposition 1.1 to the $\alpha$-stable case, $\alpha<2$. Namely, if $X$ and $u$ are related via (1.4), then under an additional assumption on $E^{*}, e_{n}(u) \geq c \cdot n^{-1 / \theta}$ for some $\theta>0$ with $1 / \theta>[1-1 / \alpha]_{+}$implies

$$
-\log \mathbb{P}\left(\|X\|_{E^{*}}<\varepsilon\right) \geq c^{\prime} \cdot \varepsilon^{-\lambda}
$$

where $1 / \lambda=1 / \theta+1 / \alpha-1$. It is very likely this is true without any additional assumption about $E^{*}$ or $E$, respectively. The answer remains open because it is closely related to the famous duality problem for entropy numbers, cf. Bourgain et al..$^{[2]}$ and Milman and Szarek $^{[12]}$. As applications of (1.5) we obtain lower estimates for the small deviation function of weighted $\alpha$-stable Levy motions, linear fractional $\alpha$-stable motions and $d$-dimensional $\alpha-$ stable Levy sheets. For a comprehensive survey of various small deviation results for stable processes, see Li and Linde ${ }^{[7]}$.

The organization of the paper is as follows. Section 2 contains a representation theorem, see (2.3), for operators $u$. This is more or less a different way to state a well-known and very useful representation theorem for stable processes as mixture of Gaussian ones. In Section 3 we compare the entropy numbers of an operator $u$ represented via (2.3) with those of the $v_{\delta}$ 's appearing there. Here we follow the ideas developed in Marcus and Pisier ${ }^{[11]}$ with refinements. Our key results are contained in Section 4. Especially, Lemma 4.2 turns out to be crucial for our later investigations. The idea is to precise an important Lemma 
of Kuelbs and $\mathrm{Li}^{[5]}$ since the original one is not sufficient for our purposes. This allows us to prove Theorem 4.5 which contains the above mentioned relation between entropy and small deviations for stable processes. Of course, Theorem 4.5 is considerably weaker than Proposition 1.1, but to our knowledge it is the first general small deviation result for nonGaussian processes. Finally, Section 5 is devoted to the problem of representing stable processes by suitable operators. As a consequence we get some applications of Theorem 4.5 for special stable processes.

Acknowledgement: The authors would like to thank the support by the RiP program in Oberwolfach in which this work originated.

\section{Integral Representation of $L_{\alpha}$-valued Operators}

The aim of this section is to transform a very useful representation theorem for stable stochastic processes into the language of linear bounded operators. Thus let $E$ be a Banach space with topological dual $E^{*}$ and let $(S, \sigma)$ be a finite measure space. All our later investigations are also valid for $\sigma$-finite measure spaces by an obvious change of density. A linear and continuous operator $u$ from $E$ into $L_{\alpha}(S, \sigma)$ for some $\alpha>0$ is said to be order bounded if

$$
\sup \left\{\int_{S} \sup _{1 \leq j \leq n}\left|u\left(x_{j}\right)(s)\right|^{\alpha} d \sigma(s): x_{j} \in E,\left\|x_{j}\right\| \leq 1\right\}<\infty .
$$

There exist useful equivalent characterizations of order bounded operators (cf. Bourbaki ${ }^{[1]}$, Vakhania el al..$^{[22]}$ and Linde $\left.{ }^{[10]}\right)$ : An operator $u$ from $E$ into $L_{\alpha}$ is order bounded iff there is a function $f \in L_{\alpha}(S, \sigma)$ such that for any $x \in E$ with $\|x\| \leq 1,|u(x)(s)| \leq f(s), \sigma-$ a.e.

Another characterization is as follows. Let $\mathcal{B}_{\sigma}\left(E^{*}\right)$ be the $\sigma$-algebra on $E^{*}$ generated by the $\sigma\left(E^{*}, E\right)$, the weak-*-open subsets of $E^{*}$. Then $u$ satisfies $(2.1)$ iff there is a function $\varphi: S \rightarrow E^{*}$, measurable with respect to $\mathcal{B}_{\sigma}\left(E^{*}\right)$ such that

$$
\int_{S}\|\varphi(s)\|_{E^{*}}^{\alpha} d \sigma(s)<\infty \quad \text { and } \quad u(x)=\langle x, \varphi\rangle, \quad x \in E
$$

We shall say that the $E^{*}$-valued function $\varphi$ decomposes $u$ weakly.

Now we state an abstract version of the representation theorem for stable processes as mixtures of Gaussian ones.

Proposition 2.1 For $0<\alpha<2$ let $u$ be an order bounded operator from $E$ into $L_{\alpha}(S, \sigma)$. Then there are probability space $(\Delta, \mathbb{Q})$ as well as (bounded) operators $v_{\delta}, \delta \in \Delta$, from a 
separable Hilbert space $H$ into $E^{*}$ such that

$$
\exp \left(-\|u(x)\|_{\alpha}^{\alpha}\right)=\int_{\Delta} \exp \left(-\frac{1}{2}\left\|v_{\delta}^{*}(x)\right\|_{H}^{2}\right) d \mathbb{Q}(\delta)
$$

for all $x \in E$.

Remark: Here and in the sequel we always regard the dual $v^{*}$ of an operator $v$ from $H$ into $E^{*}$ as a mapping from $E$ into $H$, i.e. we restrict $v^{*}$ to $E \subseteq E^{* *}$.

Proof: Let $\varphi$ be the $\mathcal{B}_{\sigma}\left(E^{*}\right)$-measurable function satisfying $(2.2)$ for $u: E \rightarrow L_{\alpha}(S, \sigma)$. Without lose of generality we can assume $u: E \rightarrow L_{\alpha}(S, \sigma)$ to be weakly decomposed by a function $\varphi: S \rightarrow E^{*}$ satisfying $\|\varphi(s)\|=1 \sigma$-a.s. and, moreover, $\sigma(S)=1$. To construct $(\Delta, \mathbb{Q})$ and operators $v_{\delta}, \delta \in \Delta$, we choose an i.i.d. sequence $\left(V_{j}\right)_{j \geq 1}$ of $S$-valued random variables possessing law $\sigma$ and an i.i.d. sequence $\left(\eta_{j}\right)_{j \geq 1}$ of standard exponential distributed random variables, independent of the $V_{j}$ 's. With these $\eta_{j}$ 's we construct dependent Gamma random variables $\Gamma_{j}:=\eta_{1}+\cdots+\eta_{j}, j \geq 1$. Let us also define the constant

$$
c_{\alpha}:=\left(\int_{0}^{\infty} x^{-\alpha} \sin x d x\right)^{1 / \alpha} \cdot\left(\mathbb{E}|\xi|^{\alpha}\right)^{-1 / \alpha}
$$

for $\xi \sim \mathcal{N}(0,1)$. Suppose that the $V_{j}$ 's and $\eta_{j}$ 's are defined on the same probability space $(\Delta, \mathbb{Q})$. If $H$ is a separable Hilbert space we choose some fixed ONB $\left(f_{j}\right)_{j \geq 1}$ in $H$ and define for each $\delta \in \Delta$ the element $v_{\delta}\left(f_{j}\right) \in E^{*}$ by

$$
v_{\delta}\left(f_{j}\right):=c_{\alpha} \cdot \Gamma_{j}(\delta)^{-1 / \alpha} \cdot \varphi\left(V_{j}(\delta)\right), \quad j=1,2, \ldots
$$

Note that the strong law of large numbers implies $\lim _{j \rightarrow \infty} \Gamma_{j} / j=1$ a.s. Hence, using the fact that $\left\|\varphi\left(V_{j}(\delta)\right)\right\|=1$ a.s., we see

$$
\sum_{j=1}^{\infty}\left\|v_{\delta}\left(f_{j}\right)\right\|^{2} \leq c_{\alpha}^{2} \cdot \sum_{j=1}^{\infty} \Gamma_{j}(\delta)^{-2 / \alpha}<\infty \quad \text { a.s. }
$$

i.e. the $v_{\delta}$ 's can be extended for almost all $\delta \in \Delta$ to bounded operators from $H$ into $E^{*}$. Let $\left(\xi_{j}\right)_{j \geq 1}$ be an i.i.d. sequence of $\mathcal{N}(0,1)$-distributed random variables, defined on $(\Omega, \mathbb{P})$ and independent of all other sequences. For $x \in E$ fixed we set

$$
\psi(\omega, \delta):=c_{\alpha} \sum_{j=1}^{\infty} \xi_{j}(\omega) \Gamma_{j}(\delta)^{-1 / \alpha}\left\langle x, \varphi\left(V_{j}(\delta)\right)\right\rangle .
$$

This sum is known, cf. Samorodnitsky and Taqqu ${ }^{[17]}$, to exist for almost all pairs of $(\omega, \delta)$ and, moreover,

$$
\mathbb{E}_{\delta} \mathbb{E}_{\omega} \exp (i \psi(\omega, \delta))=\exp \left(-\mathbb{E}_{\delta}\left|\left\langle x, \varphi\left(V_{1}(\delta)\right)\right\rangle\right|^{\alpha}\right)=\exp \left(-\|u(x)\|_{\alpha}^{\alpha}\right)
$$


On the other hand,

$$
\mathbb{E}_{\omega} \exp (i \psi(\omega, \delta))=\exp \left(-\frac{1}{2} c_{\alpha}^{2} \sum_{j=1}^{\infty} \Gamma(\delta)^{-2 / \alpha}\left|\left\langle x, \varphi\left(V_{j}(\delta)\right)\right\rangle\right|^{2}\right)=\exp \left(-\frac{1}{2}\left\|v_{\delta}^{*} x\right\|_{H}^{2}\right)
$$

which by (2.4) implies

$$
\exp \left(-\|u(x)\|_{\alpha}^{\alpha}\right)=\mathbb{E}_{\delta} \exp \left(-\frac{1}{2}\left\|v_{\delta}^{*} x\right\|_{H}^{2}\right)
$$

for all $x \in E$. This completes the proof.

Remark: By the construction of the operators $v_{\delta}$ the following is valid: Suppose that the decomposing function $\varphi$ of $u$ attains a.s. values in a (closed) subspace $F \subseteq E^{*}$. Then there are operators $v_{\delta}$ from $H$ into $F$ satisfying (2.3). A consequence of this observation is the following formulation.

Corollary 2.2 Suppose that $u$ maps from the dual space $E^{*}$ into $L_{\alpha}(S, \sigma), 0<\alpha<2$, and that $u$ is decomposed by a function $\varphi: S \rightarrow E$ with $s \rightarrow\left\langle\varphi(s), x^{*}\right\rangle$ measurable for each $x^{*} \in E^{*}$ and satisfying $\int_{S}\|\varphi(s)\|_{E}^{\alpha} d \sigma(s)<\infty$. Then there are operators $v_{\delta}: H \rightarrow E$ such that

$$
\exp \left(-\left\|u\left(x^{*}\right)\right\|_{\alpha}^{\alpha}\right)=\int_{\Delta} \exp \left(-\frac{1}{2}\left\|v_{\delta}^{*} x^{*}\right\|_{H}^{2}\right) d \mathbb{Q}(\delta)
$$

for all $x^{*} \in E^{*}$.

\section{Entropy Estimates for $L_{\alpha}-$ valued operators}

Suppose an operator $u$ from $E$ into some $L_{\alpha}$ admits representation (2.3) for suitable operators $v_{\delta}: H \rightarrow E^{*}, \delta \in \Delta$. Then this implies, cf. Marcus and Pisier ${ }^{[11]}$, that for each $x \in E$ the random variable $\delta \rightarrow 2^{-1}\left\|v_{\delta}^{*} x\right\|_{H}^{2} /\|u(x)\|_{\alpha}^{2}$ is $\alpha / 2$-stable and non-negative. In particular, for any $\varepsilon>0$ we have

$$
\mathbb{Q}\left(\delta \in \Delta: \frac{\left\|v_{\delta}^{*} x\right\|_{H}^{2}}{\|u(x)\|_{\alpha}^{2}} \leq \varepsilon\right) \leq \exp \left(-A \cdot \varepsilon^{-\alpha /(2-\alpha)}\right)
$$

for some universal constant $A>0$.

The next proposition is a slightly improved version of a result in Marcus and Pisier ${ }^{[11]}$.

Proposition 3.1 Suppose that $u: E \rightarrow L_{\alpha}$ and $v_{\delta}: H \rightarrow E^{*}, \delta \in \Delta$, are given in the representation (2.3). With $A>0$ from (3.1), set

$$
\rho_{0}:=2^{-1} \cdot(A / 3)^{1 / \alpha-1 / 2}
$$


and for $m \in \mathbb{N}$ define $\Delta_{m} \subseteq \Delta$ by

$$
\Delta_{m}:=\left\{\delta \in \Delta: e_{n}\left(v_{\delta}^{*}\right) \geq \rho_{0} \frac{e_{n}(u)}{n^{1 / \alpha-1 / 2}}, n \geq m\right\} .
$$

Then there is a universal constant $\kappa>0$ such that $\mathbb{Q}\left(\Delta_{m}\right) \geq 1-\kappa \cdot e^{-m}$ for all $m \in \mathbb{N}$.

Proof: Given $x, y \in E$ with $\|x\| \leq 1,\|y\| \leq 1$ and $\varepsilon>0$ we define

$$
D_{\varepsilon}(x, y):=\left\{\delta \in \Delta: \frac{\left\|v_{\delta}^{*}(x-y)\right\|_{H}}{\|u(x-y)\|_{\alpha}} \leq \varepsilon\right\},
$$

and obtain by (3.1) the estimate

$$
\mathbb{Q}\left(D_{\varepsilon}(x, y)\right) \leq \exp \left(-A \cdot \varepsilon^{-2 \alpha /(2-\alpha)}\right)
$$

Fix $n \in \mathbb{N}$ for now and set $\eta:=e_{n}(u)$. Then there are $2^{n-1}$ elements $x_{1}, \ldots, x_{2^{n-1}}$ in the unit ball of $E$ for which

$$
\left\|u\left(x_{i}\right)-u\left(x_{j}\right)\right\|_{\alpha} \geq \eta / 2, \quad i \neq j .
$$

Consequently, if

$$
D_{\varepsilon}(n):=\bigcup_{i, j=1}^{2^{n-1}} D_{\varepsilon}\left(x_{i}, x_{j}\right)
$$

then from (3.4) we obtain

$$
\mathbb{Q}\left(D_{\varepsilon}(n)\right) \leq 2^{2 n-2} \cdot e^{-A \cdot \varepsilon^{2 \alpha /(2-\alpha)}} .
$$

For $\delta \notin D_{\varepsilon}(n)$ it follows that

$$
\left\|v_{\delta}^{*}\left(x_{i}\right)-v_{\delta}^{*}\left(x_{j}\right)\right\|_{H} \geq \varepsilon \eta / 2, \quad i \neq j
$$

and hence

$$
e_{n}\left(v_{\delta}^{*}\right) \geq \varepsilon \eta / 2=2^{-1} \varepsilon \cdot e_{n}(u)
$$

for those $\delta$ 's. Next we apply this construction to each $n \in \mathbb{N}$ with $\varepsilon=\varepsilon_{n}$ depending on $n$ as follows:

$$
\varepsilon_{n}:=(3 n / A)^{1 / 2-1 / \alpha}
$$

where $A>0$ is as in (3.1) or (3.5). Plugging these $\varepsilon_{n}$ 's into (3.5) leads to

$$
\mathbb{Q}\left(D_{\varepsilon_{n}}(n)\right) \leq 2^{2 n} \cdot e^{-3 n} \leq e^{-n}
$$

and, furthermore, if $\delta \notin D_{\varepsilon_{n}}(n)$, then by (3.6), (3.7) and (3.2) we obtain

$$
e_{n}\left(v_{\delta}^{*}\right) \geq \rho_{0} \cdot \frac{e_{n}(u)}{n^{1 / \alpha-1 / 2}} .
$$


In other words, for $\Delta_{m}$ defined in (3.3) we have $\bigcap_{n \geq m} D_{\varepsilon_{n}}(n)^{c} \subseteq \Delta_{m}$, which by (3.8) implies

$$
\mathbb{Q}\left(\Delta_{m}\right) \geq 1-\sum_{n=m}^{\infty} e^{-n}=1-\frac{e}{e-1} \cdot e^{-m}
$$

as asserted.

The preceding Proposition tells us that certain lower estimates of $e_{n}(u)$ lead for almost all $\delta$ 's to lower estimates of $e_{n}\left(v_{\delta}^{*}\right)$. However this is not sufficient for our purposes because we have to know lower estimates for $e_{n}\left(v_{\delta}\right)$, not for $e_{n}\left(v_{\delta}^{*}\right)$. To overcome this difficulty, we have to use the strongest known form of duality results. To be more precise, let $F$ be a Banach space. We shall say that it possesses property $\mathrm{D}$ if there are constants $a, b>0$ such that for all compact operators $v$ from a Hilbert space $H$ into $F$ the estimate

$$
e_{[a \cdot n]}\left(v^{*}\right) \leq b \cdot e_{n}(v)
$$

is valid. It is an long standing open problem whether or not every Banach space $F$ possesses property D. As shown in Pajor and Tomczak-Jaegermann ${ }^{[14]}$, Thm. 3.3, spaces of so-called weak cotype 2 have this property, in particular, all $L_{p}$-spaces with $1 \leq p \leq 2$.

Corollary 3.2 Let $u: E \rightarrow L_{\alpha}$ and $v_{\delta}, \delta \in \Delta$ be given in (2.3) and suppose that almost all $v_{\delta}$ 's, mapping $H$ into a subspace $F \subseteq E^{*}$, possessing property $D$. If

$$
e_{n}(u) \geq c_{1} \cdot n^{-1 / \gamma}(\log n)^{\beta}
$$

for certain $\gamma>0$ and $\beta \in \mathbb{R}$, then there are constants $\rho, \kappa, a>0$ such that for all $m \in \mathbb{N}$

$$
\mathbb{Q}\left(\delta \in \Delta: e_{n}\left(v_{\delta}\right) \geq \rho \cdot n^{-1 / \gamma-1 / \alpha+1 / 2}(\log n)^{\beta}, n \geq m\right) \geq 1-\kappa e^{-a m}
$$

Proof: ¿From Proposition 3.1 and (3.10) we derive

$$
\mathbb{Q}\left(\delta \in \Delta: e_{n}\left(v_{\delta}^{*}\right) \geq \rho_{0} \cdot c_{1} \cdot n^{-1 / \gamma-1 / \alpha+1 / 2}(\log n)^{\beta}, n \geq m\right) \geq 1-\kappa e^{-m},
$$

and by (3.9) this implies

$$
\mathbb{Q}\left(\delta \in \Delta: e_{n}\left(v_{\delta}\right) \geq \rho \cdot n^{-1 / \gamma-1 / \alpha+1 / 2}(\log n)^{\beta}, n \geq \frac{m-1}{a}\right) \geq 1-\kappa e^{-m}
$$

where $a>0$ is as in (3.10) and $\rho>0$ depends on $c_{1}, \gamma, \beta$ and $a, b$ from (3.9). Changing $\kappa$ in (3.12) suitably, (3.11) follows by direct calculations. 


\section{Small Ball Estimates of Stable Measures}

Let $(K, d)$ be a precompact metric space and denote by $e_{n}(K)=e_{n}(K, d)$ the dyadic entropy numbers of $K$, i.e. $e_{n}(K)$ is the minimal $\varepsilon>0$ for which there exists an $\varepsilon$-cover of $K$ with at most $2^{n-1}$ elements in $K$. In certain situations it is easier to work with the metric entropy $H(\varepsilon, K)=\log N(\varepsilon, K)$ of $K$, where

$$
N(\varepsilon, K):=\inf \{n \in \mathbb{N}: \exists \varepsilon-\text { cover of cardinality less than } n \text { of } K\}
$$

The metric entropy function $H(\varepsilon, K)$ may be regarded as a inverse to that of entropy numbers. To be more precise and make it applicable to random setting, we need the following lemma with relevant constants expressed explicitly.

Lemma 4.1 Let $(K, d)$ be a metric space such that for certain $\gamma>0$ and $\beta \in \mathbb{R}$ holds

$$
e_{n}(K) \geq c_{1} \cdot n^{-1 / \gamma}(\log n)^{\beta}
$$

whenever $n \geq m$ for some $m \in \mathbb{N}$. Then this yields

$$
H(\varepsilon, K) \geq c_{2} \cdot \varepsilon^{-\gamma} \log (1 / \varepsilon)^{\beta \gamma}
$$

provided that $\varepsilon<\varepsilon_{0}=\varepsilon_{0}(m)$ with

$$
\varepsilon_{0}:=c_{1} \cdot m^{-1 / \gamma}(\log m)^{\beta}
$$

The constant $c_{2}>0$ in (4.2) depends only on $c_{1}>0$ of (4.1) and on $\gamma$ and $\beta$.

Proof: Of course, it suffices to prove this for $m$ sufficiently large. Hence we may assume that $n \rightarrow n^{-1 / \gamma}(\log n)^{\beta}$ is decreasing for $n \geq m$. Consequently, for $\varepsilon_{0}$ defined by (4.3) and $\varepsilon<\varepsilon_{0}$ we find a unique $n \geq m$ such that with $c_{1}>0$ as in (4.1)

$$
c_{1} \cdot(n+1)^{-1 / \gamma}(\log (n+1))^{\beta} \leq \varepsilon<c_{1} \cdot n^{-1 / \gamma}(\log n)^{\beta}
$$

Thus by (4.1) we obtain $e_{n}(K)>\varepsilon$, i.e. it holds $N(\varepsilon, K) \geq 2^{n-1}$ and using (4.4) this leads to

$$
H(\varepsilon, K) \geq(n-1) \log 2 \geq \frac{\log 2}{2} \cdot c_{1}^{\gamma} \cdot \varepsilon^{-\gamma}(\log (n+1))^{\beta \gamma} .
$$

Our lemma then follows by using (4.4) and some simple calculation.

Before proceeding further let us recall some basic facts about centered Gaussian measures and the operators generating them. If $\nu$ is a probability measure on the weak-*-Borel sets 
$\mathcal{B}_{\sigma}\left(E^{*}\right)$ of $E^{*}$, then its characteristic function $\hat{\nu}$ is a mapping from $E$ into the complex numbers defined by

$$
\hat{\nu}(x):=\int_{E^{*}} \exp \left(i\left\langle x, x^{*}\right\rangle\right) d \nu\left(x^{*}\right), \quad x \in E .
$$

The measure $\nu$ is said to be centered Gaussian if there are a separable Hilbert space $H$ and an operator $v$ mapping $H$ into $E^{*}$ such that

$$
\hat{\nu}(x)=\exp \left(-\frac{1}{2}\left\|v^{*}(x)\right\|^{2}\right)
$$

for all $x \in E$. Denote by $K_{\nu} \subseteq E^{*}$ the unit ball of the reproducing kernel Hilbert space of $\nu$, which coincides with $\{v(h): h \in H,\|h\| \leq 1\}$ in our situation, i.e. for $\nu$ generated by $v$ via (4.7). The set $K_{\nu}$ is known to be precompact with respect to the norm topology on $E^{*}$. By Sudakov's Theorem ${ }^{[19]}$ it even holds $\sup _{n \geq 1} n^{1 / 2} e_{n}\left(K_{\nu}\right)<\infty$. For a probability measure $\nu$ on $\mathcal{B}_{\sigma}\left(E^{*}\right)$ we define its small ball function $\phi_{\nu}$ at the log-level by

$$
\phi_{\nu}(\varepsilon):=-\log \nu\left\{x^{*} \in E^{*}:\left\|x^{*}\right\| \leq \varepsilon\right\} .
$$

As discovered in Kuelbs and $\mathrm{Li}^{[5]}$ for Gaussian $\nu$ the function $\phi_{\nu}$ is tightly related with the degree of compactness of $K_{\nu}$. One of the basic estimates in the paper asserts

$$
H\left(\frac{\varepsilon}{\lambda}, K_{\nu}\right) \leq \frac{\lambda^{2}}{2}+\phi_{\nu}(\varepsilon)
$$

for all $\varepsilon, \lambda>0$. Note that we investigate measures $\nu$ on $\mathcal{B}_{\sigma}\left(E^{*}\right)$ here and it may happen, even in the Gaussian case, that $\phi_{\nu}(\varepsilon)=\infty$ for all $\varepsilon<\varepsilon_{0}$. In order to be applicable to random setting, we need the following lemma based on (4.9) that expresses relevant constants explicitly.

Lemma 4.2 Let $\nu$ on $\mathcal{B}_{\sigma}\left(E^{*}\right)$ be generated by $v$ via (4.7) and suppose that for some $\gamma \in(0,2)$ and $\beta \in \mathbb{R}$ we have

$$
H\left(\varepsilon, K_{\nu}\right) \geq c_{2} \cdot \varepsilon^{-\gamma} \log (1 / \varepsilon)^{\beta \gamma}
$$

provided that $\varepsilon<\varepsilon_{0}$ for a certain $\varepsilon_{0}>0$. Then this implies

$$
\phi_{\nu}(\varepsilon) \geq c_{3} \cdot \varepsilon^{-2 \gamma /(2-\gamma)} \log (1 / \varepsilon)^{2 \beta \gamma /(2-\gamma)}
$$

for all $\varepsilon<\varepsilon_{1}$ where

$$
\varepsilon_{1}=c_{4} \cdot \varepsilon_{0}^{1-\gamma / 2} \log \left(1 / \varepsilon_{0}\right)^{\beta \gamma / 2} .
$$

Here the constants $c_{3}, c_{4}>0$ in (4.11) and (4.12) depend only on $c_{2}, \gamma$ and $\beta$. 
Proof: In view of (4.10), the basic estimate (4.9) leads to

$$
c_{2} \cdot\left(\frac{\varepsilon}{\lambda}\right)^{-\gamma} \cdot \log \left(\frac{\lambda}{\varepsilon}\right)^{\beta \gamma} \leq \frac{\lambda^{2}}{2}+\phi_{\nu}(\varepsilon)
$$

provided that $\varepsilon / \lambda<\varepsilon_{0}$. We define now a constant $a \in(0,1]$ as follows: If $\beta>0$, let $a=1$, while for $\beta<0$ we choose $a>0$ small so that

$$
a^{\gamma} \cdot\left(\frac{3}{2-\gamma}\right)^{\beta \gamma}-\frac{a^{2}}{2}:=a^{\prime}>0 .
$$

Recall that we have $0<\gamma<2$. With this number $a$ and with $c_{2}>0$ in (4.10) we apply (4.13) for

$$
\lambda:=a \cdot c_{2}^{1 /(2-\gamma)} \cdot \varepsilon^{-\gamma /(2-\gamma)} \cdot \log (1 / \varepsilon)^{\beta \gamma /(2-\gamma)} .
$$

The remaining step is to treat the log-term appearing on the left hand side of (4.13) with $\lambda$ given in (4.15). First note that it suffices to prove the Lemma for $\varepsilon_{0}$ sufficiently small. Hence, if $\beta>0$ we have by using $2 /(2-\gamma)>1$

$$
\log (\lambda / \varepsilon) \geq \log (1 / \varepsilon)
$$

for $\varepsilon<\varepsilon_{0}$. If $\beta<0$, the constant $a$ is less than 1 , and hence

$$
\log (\lambda / \varepsilon) \leq \frac{2}{2-\gamma} \log (1 / \varepsilon)+\frac{\log c_{2}}{2-\gamma} \leq \frac{3}{2-\gamma} \log (1 / \varepsilon)
$$

again for $\varepsilon_{0}$ sufficiently small. Consequently, (4.16) and (4.17) lead to

$$
\begin{aligned}
& \log (\lambda / \varepsilon)^{\beta \gamma} \geq \log (1 / \varepsilon)^{\beta \gamma}, \quad \beta>0, \quad \text { and } \\
& \log (\lambda / \varepsilon)^{\beta \gamma} \geq\left(\frac{3}{2-\gamma}\right)^{\beta \gamma} \cdot \log (1 / \varepsilon)^{\beta \gamma}, \quad \beta<0
\end{aligned}
$$

Combining this with (4.13) and (4.14) yields, in dependence of $\beta<0$ or $\beta>0$, respectively, that

$$
\begin{aligned}
& \phi_{\nu}(\varepsilon) \geq\left[a^{\gamma}\left(\frac{3}{2-\gamma}\right)^{\beta \gamma}-\frac{a^{2}}{2}\right] \cdot c_{2}^{2 /(2-\gamma)} \cdot \varepsilon^{-2 \gamma /(2-\gamma)} \cdot \log (1 / \varepsilon)^{2 \beta \gamma /(2-\gamma)} \\
& \phi_{\nu}(\varepsilon) \geq \frac{1}{2} \cdot c_{2}^{2 /(2-\gamma)} \cdot \varepsilon^{-2 \gamma /(2-\gamma)} \cdot \log (1 / \varepsilon)^{2 \beta \gamma /(2-\gamma)}
\end{aligned}
$$

This proves (4.11) with $c_{3}=a^{\prime} \cdot c_{2}^{2 /(2-\gamma)}$ for $\beta<0$ and with $c_{3}=c_{2}^{2 /(2-\gamma)} / 2$ if $\beta>0$.

Finally observe that (4.18) or (4.19) only hold under the assumption $\varepsilon / \lambda<\varepsilon_{0}$ with $\lambda=\lambda(\varepsilon)$ defined in (4.15). It is easy to see that $\varepsilon / \lambda$ admits two-sided estimates with multiples of $\varepsilon^{2 /(2-\gamma)} \cdot \log (1 / \varepsilon)^{-\beta \gamma /(2-\gamma)}$ where the appearing constants only depend on $c_{2}, \beta$ 
and $\gamma$. Consequently, there is some $c_{4}>0$ depending only on these numbers such that with $\varepsilon_{1}:=c_{4} \cdot \varepsilon_{0}^{(2-\gamma) / 2} \cdot \log \left(1 / \varepsilon_{0}\right)^{\beta \gamma / 2}$ the estimate $\varepsilon<\varepsilon_{1}$ always yields $\varepsilon / \lambda<\varepsilon_{0}$. This completes the proof.

Combining Lemma 4.1 with Lemma 4.2 leads to the following.

Proposition 4.3 Let $\nu$ and $K_{\nu}$ be as before and suppose that for all $n \geq m$ we have

$$
e_{n}\left(K_{\nu}\right) \geq c_{1} \cdot n^{-1 / \gamma}(\log n)^{\beta}
$$

for some $c_{1}>0$, some $\gamma \in(0,2)$ and $\beta \in \mathbb{R}$. Then

$$
\phi_{\nu}(\varepsilon) \geq c_{3} \cdot \varepsilon^{-2 \gamma /(2-\gamma)} \cdot \log (1 / \varepsilon)^{2 \beta \gamma /(2-\gamma)}
$$

for all $\varepsilon<\varepsilon_{1}=\varepsilon_{1}(m)$ where

$$
\varepsilon_{1}:=c_{4} \cdot m^{-1 / \gamma+1 / 2} \cdot(\log m)^{\beta}
$$

and $c_{3}, c_{4}>0$ depend only on $c_{1}, \gamma$ and $\beta$.

Before stating and proving the main result of this paper let us recall some facts about symmetric $\alpha$-stable $(\mathrm{S} \alpha \mathrm{S})$ measures. A probability measure $\mu$ on $\mathcal{B}_{\sigma}\left(E^{*}\right)$ is said to be $\mathrm{S} \alpha \mathrm{S}$, $0<\alpha \leq 2$, if there is some operator $u: E \rightarrow L_{\alpha}(S, \sigma)$ for some finite measure space $(S, \sigma)$ such that the characteristic function $\hat{\mu}$ of $\mu$ can be written as

$$
\hat{\mu}(x)=\exp \left(-\|u(x)\|_{\alpha}^{\alpha}\right), \quad x \in E .
$$

We are mainly interested in the non-Gaussian case, i.e. we suppose $0<\alpha<2$. Since $\mu$ is Radon on the locally convex space $\left[E^{*}, \sigma\left(E^{*}, E\right)\right]$, by Tortrat's theorem ${ }^{[21]}$, we can always assume $u$ to be weakly decomposed by an $\alpha$-integrable $E^{*}$-valued function. Consequently, according to Proposition 2.1 there exist a probability space $(\Delta, \mathbb{Q})$ and operators $v_{\delta}: H \rightarrow$ $E^{*}, \delta \in \Delta$, such that

$$
\hat{\mu}(x)=\exp \left(-\|u(x)\|_{\alpha}^{\alpha}\right)=\int_{\Delta} e^{-\frac{1}{2}\left\|v_{\delta}^{*} x\right\|_{H}^{2}} d \mathbb{Q}(\delta)
$$

for $x \in E$.

The next result asserts that for $\mathbb{Q}$-almost all $\delta \in \Delta$ the function $x \rightarrow \exp \left(-\left\|v_{\delta}^{*} x\right\|_{H}^{2} / 2\right)$ appearing at the right hand side of (4.23) is characteristic function of a $\sigma\left(E^{*}, E\right)$-Radon (Gaussian) measure on $E^{*}$. For a proof we refer to Sztencel ${ }^{[18]}$. 
Proposition 4.4 If $\mu$ and the $v_{\delta}$ 's satisfy (4.23), then for almost all $\delta \in \Delta$ there exist centered Gaussian measures $\nu_{\delta}$ on $\mathcal{B}_{\sigma}\left(E^{*}\right)$ satisfying

$$
\widehat{\nu_{\delta}}(x)=\exp \left(-\frac{1}{2}\left\|v_{\delta}^{*} x\right\|_{H}^{2}\right), \quad x \in E .
$$

Moreover, for any $B \in \mathcal{B}_{\sigma}\left(E^{*}\right)$

$$
\mu(B)=\int_{\Delta} \nu_{\delta}(B) d \mathbb{Q}(\delta)
$$

Our next objective is to prove the main result of the present paper. It relates the behavior of the small ball function $\phi_{\mu}$ for an $\mathrm{S} \alpha \mathrm{S}$ measure $\mu$ on $E^{*}$ with the degree of compactness of $u: E \rightarrow L_{\alpha}$. Here $\mu$ and $u$ are related via (4.22). Let us also mention that we necessarily have $e_{n}\left(u_{1}\right)=e_{n}\left(u_{2}\right)$ for two operators $u_{1}, u_{2}$ both related to $\mu$ by (4.22).

Theorem 4.5 Let $E$ be a Banach space for which $E^{*}$ possesses property $D$ and let $\mu$ be an $S \alpha S$ measure on $\mathcal{B}_{\sigma}\left(E^{*}\right)$ with characteristic function represented by an operator $u: E \rightarrow L_{\alpha}$. If $u$ satisfies

$$
\liminf _{n \rightarrow \infty} n^{1 / \theta} \cdot(\log n)^{-\beta} \cdot e_{n}(u) \geq C>0
$$

for some $\theta>0$ with $1 / \theta>[1-1 / \alpha]_{+}$and some $\beta \in \mathbb{R}$, then

$$
\liminf _{\varepsilon \rightarrow 0} \varepsilon^{\lambda} \cdot \log (1 / \varepsilon)^{-\beta \lambda} \cdot \phi_{\mu}(\varepsilon) \geq c_{0} \cdot C^{\lambda}>0
$$

with $1 / \lambda=1 / \theta+1 / \alpha-1$ and some universal $c_{0}>0$ depending only on $\alpha, \theta, \beta$ and the constants $a, b>0$ appearing in (3.9).

Proof: We first suppose

$$
e_{n}(u) \geq n^{-1 / \theta}(\log n)^{\beta}
$$

whenever $n \geq n_{0}$ for a certain $n_{0} \in \mathbb{N}$. Let $\nu_{\delta}, \delta \in \Delta$, be centered Gaussian measures on $\mathcal{B}_{\sigma}\left(E^{*}\right)$ related to $\mu$ as in (4.24) and possessing characteristic functions

$$
\widehat{\nu_{\delta}}(x)=\exp \left(-\frac{1}{2}\left\|v_{\delta}^{*} x\right\|_{H}^{2}\right), \quad x \in E,
$$

for some operators $v_{\delta}: H \rightarrow E^{*}$. An application of Corollary 3.2 leads in view of (4.27) to the following: There exist constants $\rho, \kappa, a>0$ such that for all $m \geq m_{0}$ it follows that

$$
\mathbb{Q}\left(\delta \in \Delta: e_{n}\left(v_{\delta}\right) \geq \rho \cdot n^{-1 / \lambda-1 / 2} \cdot(\log n)^{\beta}, \quad n \geq m\right) \geq 1-\kappa \cdot e^{-a m} .
$$

Next we apply Proposition 4.3 with $c_{1}=\rho$ from (4.28) and with $\gamma \in(0,2)$ defined by $1 / \gamma:=1 / \lambda+1 / 2$. Then for $\varepsilon>0$ small there are integers $m_{\varepsilon} \in \mathbb{N}$ satisfying

$$
\varepsilon \approx m_{\varepsilon}^{-1 / \lambda} \cdot\left(\log m_{\varepsilon}\right)^{\beta}
$$


such that, if

$$
e_{n}\left(K_{\nu}\right) \geq \rho \cdot n^{-1 / \lambda-1 / 2} \cdot(\log n)^{\beta}
$$

for all $n \geq m_{\varepsilon}$, then

$$
\phi_{\nu}(\varepsilon) \geq c_{3} \cdot \varepsilon^{-\lambda} \cdot \log (1 / \varepsilon)^{\beta \lambda} .
$$

Now we apply this for $\nu=\nu_{\delta}$ with $\delta$ 's satisfying the condition in (4.28). Observe that $e_{n}\left(K_{\nu_{\delta}}\right)=e_{n}\left(v_{\delta}\right)$. Thus, if we define for $c_{3}>0$ given in (4.30) the set

$$
\Delta(\varepsilon):=\left\{\delta \in \Delta: \phi_{\nu_{\delta}}(\varepsilon) \geq c_{3} \cdot \varepsilon^{-\lambda} \cdot \log (1 / \varepsilon)^{\beta \lambda}\right\}
$$

then we get

$$
\mathbb{Q}(\Delta(\varepsilon)) \geq 1-\kappa \cdot e^{-a m_{\varepsilon}}
$$

for every small $\varepsilon>0$. Since (4.29) is equivalent to $m_{\varepsilon} \approx \varepsilon^{-\lambda} \cdot \log (1 / \varepsilon)^{\beta \lambda}$ we finally obtain for a certain $\tilde{c}>0$,

$$
\mathbb{Q}(\Delta(\varepsilon)) \geq 1-\kappa \cdot e^{-\tilde{c} \varepsilon^{-\lambda} \log (1 / \varepsilon)^{\beta \lambda}} .
$$

With this preparation we are now in position to prove (4.26) under the special assumption (4.27). ¿From (4.33) and (4.31) we derive

$$
\begin{aligned}
\mu\left(\left\|x^{*}\right\|<\varepsilon\right) & =\int_{\Delta} \nu_{\delta}\left(\left\|x^{*}\right\|<\varepsilon\right) d \mathbb{Q}(\delta) \\
& \leq \int_{\Delta(\varepsilon)} \exp \left(-\phi_{\nu_{\delta}}(\varepsilon)\right) d \mathbb{Q}(\delta)+\mathbb{Q}\left(\Delta(\varepsilon)^{c}\right) \\
& \leq \exp \left(-c_{3} \cdot \varepsilon^{-\lambda} \cdot \log (1 / \varepsilon)^{\beta \lambda}\right)+\kappa \cdot \exp \left(-\tilde{c} \cdot \varepsilon^{-\lambda} \cdot \log (1 / \varepsilon)^{\beta \lambda}\right) .
\end{aligned}
$$

Consequently, there is some constant $c_{0}>0$ such that (4.27) implies

$$
\phi_{\mu}(\varepsilon) \geq c_{0} \cdot \varepsilon^{-\lambda} \cdot \log (1 / \varepsilon)^{\beta \lambda}
$$

for small $\varepsilon>0$. In particular, it follows that

$$
\liminf _{\varepsilon \rightarrow 0} \varepsilon^{\lambda} \cdot \log (1 / \varepsilon)^{-\beta \lambda} \cdot \phi_{\mu}(\varepsilon) \geq c_{0}
$$

Here the constant $c_{0}>0$ in (4.34) or (4.35), is, of course, independent of the operator $u$ (or, equivalently, of the corresponding measure $\mu$ ).

As the final step, we remove the condition (4.27). Assume (4.25) and let $\mu_{0}$ be defined by

$$
\widehat{\mu_{0}}(x):=\exp \left(-\|u(x) / C\|_{\alpha}^{\alpha}\right), \quad x \in E .
$$


Then condition (4.27) is satisfied and hence

$$
\liminf _{\varepsilon \rightarrow 0} \varepsilon^{\lambda} \cdot \log (1 / \varepsilon)^{-\beta \lambda} \cdot \phi_{\mu_{0}}(\varepsilon) \geq c_{0} .
$$

By noticing $\phi_{\mu_{0}}(\varepsilon)=-\log \mu\left(\left\|x^{*}\right\|<\varepsilon C\right)=\phi_{\mu}(\varepsilon C)$, we complete the proof.

There are several remarks to Theorem 4.5. First, suppose that almost all measures $\nu_{\delta}$ are concentrated on a subspace $F \subseteq E^{*}$ (or, equivalently, almost all operators $v_{\delta}$ map into $F)$. Then for the validity of Theorem 4.5 it suffices that $F$ possesses property D. Second, the restriction $1 / \theta>1-1 / \alpha$ for $1<\alpha<2$ is natural due to a lower entropy estimate for bounded S $\alpha$ S-processes, see, Marcus and Pisier ${ }^{[11]}$ or Samorodnitsky and Taqqu ${ }^{[17]}$, Thm. 12.3.1. Finally, as shown in Ryznar ${ }^{[15]}$, for $0<\alpha<1$ and all $\mathrm{S} \alpha \mathrm{S}-$ measures $\mu$,

$$
\phi_{\mu}(\varepsilon) \leq c \cdot \varepsilon^{-\alpha /(1-\alpha)}, \quad 0<\varepsilon<1
$$

This explains why the $\lambda$ appearing in (4.26) always satisfy $\lambda<\alpha /(1-\alpha)$.

\section{$5 \quad$ Examples and Applications}

A stochastic process $X=(X(t))_{t \in T}$ over an index set $T \neq \emptyset$ is said to be S $\alpha \mathrm{S}$ for some $\alpha \in(0,2]$ if for all $t_{1}, \ldots, t_{n} \in T$ and all real numbers $\lambda_{1}, \ldots, \lambda_{n}$, the real random variable $\sum_{j=1}^{n} \lambda_{j} X\left(t_{j}\right)$ is $\mathrm{S} \alpha \mathrm{S}$-distributed.

We shall restrict ourselves to $\mathrm{S} \alpha \mathrm{S}$-processes possessing a version (the finite dimensional distributions coincide) which admits an integral representation in the sense of Ch. 13 in Samorodnitsky and Taqqu ${ }^{[17]}$. In other words, we investigate S $\alpha$ S-processes $X$ for which there exist a finite measure space $(S, \sigma)$ and a kernel $K: T \times S \rightarrow \mathbb{R}$ such that for each $t \in T$ the function $s \rightarrow K(t, s)$ is measurable with

$$
\int_{S}|K(t, s)|^{\alpha} d \sigma(s)<\infty
$$

and for all $\lambda_{1}, \ldots, \lambda_{n} \in \mathbb{R}$ and all $t_{1}, \ldots, t_{n} \in T$ we have

$$
\mathbb{E} \exp \left(i \sum_{j=1}^{n} \lambda_{j} X\left(t_{j}\right)\right)=\exp \left(-\int_{S}\left|\sum_{j=1}^{n} \lambda_{j} K\left(t_{j}, s\right)\right|^{\alpha} d \sigma(s)\right) .
$$

The class of those $\mathrm{S} \alpha \mathrm{S}$-processes is very broad and contains all special processes of interest such as those processes with property $S$ given in Samorodnitsky and Taqqu ${ }^{[17]}$.

Let $X$ be an $\mathrm{S} \alpha \mathrm{S}$-process generated by $K: T \times S \rightarrow \mathbb{R}$ via (5.1). Our aim is to construct a suitable $L_{\alpha}$-valued operator $u$ tightly related to $X$. This allows us to transform entropy estimates of $u$ into those of sets generated by $X$ and vice versa. 
As a first example of such a construction we investigate bounded processes. Suppose that $X$ has a bounded version, i.e. there is a version $\tilde{X}$ of $X$, defined on $\tilde{\Omega}$, such that for each $\tilde{\omega} \in \tilde{\Omega}$ we have

$$
\sup _{t \in T}|\tilde{X}(t)(\tilde{\omega})|<\infty
$$

If $K$ is the corresponding kernel to $X$ (or $\tilde{X}$ ) by Thm. 10.2.3 in Samorodnitsky and Taqqu ${ }^{[17]}$ it follows that

$$
\sup \left\{\int_{S} \sup _{t \in T_{0}}|K(t, s)|^{\alpha} d \sigma(s): T_{0} \subset T \text { countable }\right\}<\infty
$$

Hence, if the Banach space $l_{1}(T)$ is defined by

$$
l_{1}(T):=\left\{x=\left(x_{t}\right)_{t \in T}: \sum_{t \in T}\left|x_{t}\right|<\infty\right\},
$$

then the operator $u$ with

$$
u(x)(s):=\sum_{t \in T} x_{t} \cdot K(t, s), \quad x=\left(x_{t}\right)_{t \in T} \in l_{1}(T),
$$

is well-defined and bounded from $l_{1}(T)$ into $L_{\alpha}(S, \sigma)$ because of (5.2). Recall that for $x \in l_{1}(T)$ at most countable many of the $x_{t}$ 's are different from zero. By similar arguments, $u$ is even order bounded. Fix now a positive number $r<\alpha$. By a well-known property of stable random variables

$$
\left(\mathbb{E}\left|\sum_{j=1}^{n} \lambda_{j} X\left(t_{j}\right)\right|^{r}\right)^{1 / r}=c_{\alpha, r} \cdot\left\|\sum_{j=1}^{n} \lambda_{j} \cdot K\left(t_{j}, \cdot\right)\right\|_{\alpha}
$$

for some universal $c_{\alpha, r}>0$. Thus, if we define

$$
s(X):=\left\{\sum_{j=1}^{n} \lambda_{j} X\left(t_{j}\right): \sum_{j=1}^{n}\left|\lambda_{j}\right| \leq 1, t_{j} \in T\right\}
$$

and regard it as subset of $L_{r}(\Omega, \mathbb{P})$, by the definition of $u$ via $(5.3)$ it easily follows that

$$
e_{n}(u)=c_{\alpha, r}^{-1} \cdot e_{n}(s(X))
$$

where the entropy on the right hand side of (5.4) is taken with respect to the $L_{r}$-distance. In other words, compactness properties of the operator $u$ are equivalent to those of the set $s(X)$, the set of symmetric convex combinations of paths of $X$. Unfortunately, it is not known whether or not the dual of $l_{1}(T)$ (space of bounded functions on $T$ ) possesses property $\mathrm{D}$, and thus in the moment we are not able to apply Theorem 4.5 here. 
Let us suppose now that there is a metric $d$ on the index set $T$ for which $(T, d)$ is separable. Define $\mathcal{B}(T)$ as $\sigma$-algebra of Borel sets (w.r.t. the topology generated by $d$ ). We assume now that the S $\alpha$ S-process $X$ has a measurable version, i.e. there is a version $\tilde{X}$ of $X$ for which $(t, \tilde{\omega}) \rightarrow \tilde{X}(t, \tilde{\omega})$ is measurable on $T \times \tilde{\Omega}$ w.r.t. the product $\sigma$-algebra. Note that by Cor. 11.1.2 in Samorodnitsky and Taqqu ${ }^{[17]}$ such a measurable version exists iff the kernel $K$ with (5.1) may be chosen measurable on $T \times S$. Next let $X$ be a measurable $\mathrm{S} \alpha \mathrm{S}$-process over $T$ and let $\rho$ be some finite measure on $(T, \mathcal{B}(T))$. Then we are interested in processes $X$ that satisfy

$$
\mathbb{P}\left(\int_{T}|X(t)|^{p} d \rho(t)<\infty\right)=1
$$

for some $p \in[1, \infty]$ with the usual modification for $p=\infty$. In particular, (5.5) implies that for each $g \in L_{q}(T, \rho), q^{-1}+p^{-1}=1$, the random variable

$$
X_{g}:=\int_{T} X(t) g(t) d \rho(t)
$$

is well-defined. In fact, it is not clear at all that $X_{g}$ is $\mathrm{S} \alpha \mathrm{S}$.

Proposition 5.1 Let $X$ be a measurable $S \alpha S$-process over a separable metric space $T$ satisfying (5.5) for some $p \in[1, \infty]$. For $g \in L_{q}(T, \rho)$ define $X_{g}$ by (5.6). Then the following are valid:

(1) The random variable $X_{g}$ is $S \alpha S$.

(2) It follows that

$$
\mathbb{E} e^{i X_{g}}=\exp \left(-\int_{S}\left|\int_{T} K(t, s) g(t) d \rho(t)\right|^{\alpha} d \sigma(s)\right) .
$$

Proof: For $g$ 's in $L_{q}(T, \rho)$ with $g \geq 0$, both assertions follow directly from Thm. 11.4.1 in Samorodnitsky and Taqqu ${ }^{[17]}$. The arguments there can be modified to treat the general case by considering the positive and negative part of $g \in L_{q}$ separately. We omit the details.

The preceding Proposition implies in particular that

$$
\int_{T} K(t, \cdot) g(t) d \rho(t) \in L_{\alpha}(S, \sigma)
$$

for each $g \in L_{q}(T, \rho)$. Hence, if

$$
(u g)(s):=\int_{T} K(t, s) g(t) d \rho(t), \quad g \in L_{q}(T, \rho),
$$

then $u$ maps $L_{q}(T, \rho)$ into $L_{\alpha}(S, \sigma)$ and it is bounded by the Closed Graph Theorem. In this notation (5.7) may now be written as

$$
\mathbb{E} \exp \left(i \int_{T} X(t) g(t) d \rho(t)\right)=\exp \left(-\|u(g)\|_{\alpha}^{\alpha}\right)
$$


Using similar arguments as in the case of bounded processes it follows

$$
e_{n}(u)=c_{\alpha, r}^{-1} \cdot e_{n}\left(s_{p}(X)\right)
$$

where

$$
s_{p}(X):=\left\{\int_{T} X(t) g(t) d \rho(t):\|g\|_{L_{q}(T, \rho)} \leq 1\right\}
$$

and the entropy numbers of $s_{p}(X)$ are taken w.r.t. $L_{r}$-norm for a certain positive $r<\alpha$.

Since for $1 \leq p \leq 2$ the space $L_{p}(T, \rho)$ possesses property D, Theorem 4.5 applies here and leads to the following.

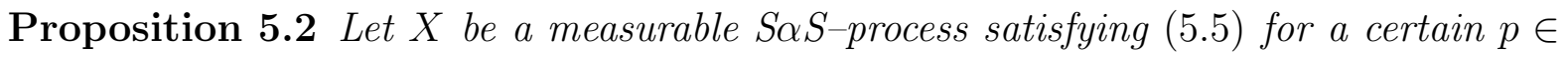
$[1,2]$. If

$$
e_{n}\left(s_{p}(X)\right) \geq c \cdot n^{-1 / \theta} \cdot(\log n)^{\beta}
$$

for some $\theta>0$ with $1 / \theta>[1-1 / \alpha]_{+}$, then

$$
-\log \mathbb{P}\left(\int_{T}|X(t)|^{p} d \rho(t)<\varepsilon^{p}\right) \geq c^{\prime} \cdot \varepsilon^{-\lambda} \cdot \log (1 / \varepsilon)^{\beta \lambda}
$$

where $1 / \lambda=1 / \theta+1 / \alpha-1$.

Remark: Note that the preceding Proposition gives also some information for the case $p=\infty$ (provided $X$ is bounded). Indeed, since $\|X\|_{p} \leq \rho(T)^{1 / p} \cdot\|X\|_{\infty}$, condition (5.10) for some $p \leq 2$ implies

$$
-\log \mathbb{P}\left(\sup _{t \in T}|X(t)|<\varepsilon\right) \geq c^{\prime} \cdot \varepsilon^{-\lambda} \cdot \log (1 / \varepsilon)^{\beta \lambda}
$$

for the same $\lambda$.

Next we present some interesting examples.

Example 1: Let $u$ from $L_{q}[0,1]$ to $L_{\alpha}[0,1]$ be defined by

$$
(u g)(s):=\int_{s}^{1} g(t) d t .
$$

This operator is tightly related with $\alpha$-Levy motion $Z_{\alpha}$ on $[0,1]$, i.e. for $g \in L_{q}[0,1]$ we have

$$
\mathbb{E} \exp \left(i \int_{0}^{1} Z_{\alpha}(t) g(t) d t\right)=\exp \left(-\|u(g)\|_{\alpha}^{\alpha}\right)
$$

Since (cf. Lifshits and Linde ${ }^{[9]}$ ) for all $p$ and $\alpha$ we have $e_{n}(u) \approx n^{-1}$, Proposition 5.2 and the following remark imply

$$
-\log \mathbb{P}\left(\left\|Z_{\alpha}\right\|_{p}<\varepsilon\right) \geq c \cdot \varepsilon^{-\alpha}
$$


for $1 \leq p \leq \infty$ and $0<\alpha<2$. This is the correct order as shown in Chen et al. ${ }^{[3]}$ and Mogulski ${ }^{[13]}$.

Example 2: Let $w:(0, \infty) \rightarrow[0, \infty)$ be a weight function such that for some $p \in[1, \infty)$ we have

$$
\mathbb{P}\left(\int_{0}^{\infty}\left|w(t) Z_{\alpha}(t)\right|^{p} d t<\infty\right)=1
$$

Here as before $\left(Z_{\alpha}(t)\right)_{t \geq 0}$ denotes a standard $\alpha$-stable Levy motion. Note that (cf. Samorodnitsky and Taqqu ${ }^{[17]}$, p. 510) we have (5.11) iff

$$
\begin{aligned}
& \int_{0}^{\infty} w(t)^{p} t^{p / \alpha} d t<\infty \quad \text { for } \quad 0<p<\alpha, \\
& \int_{0}^{\infty}\left[\int_{s}^{\infty} w(t)^{p} d t\right]^{\alpha / p} d s<\infty \quad \text { for } \quad 0<\alpha<p
\end{aligned}
$$

In the case $\alpha=p$ property (5.11) is satisfied iff

$$
\int_{0}^{\infty} t w(t)^{p} d t<\infty
$$

and, moreover,

$$
\int_{0}^{\infty} \int_{s}^{\infty} w(t)^{p} \cdot \log _{+}\left(t \cdot \int_{s}^{\infty} w(x)^{p} d x\right)^{-1} d t d s<\infty
$$

A corresponding operator $u: L_{q}(0, \infty) \rightarrow L_{\alpha}(0, \infty)$ may be defined by

$$
(u f)(s)=\int_{s}^{\infty} w(t) \cdot f(t) d t
$$

It was shown in Lifshits and Linde ${ }^{[9]}$ that (for $\alpha \geq 1$ ) the operator $u$ defined by (5.12) admits the following lower entropy estimate:

$$
\liminf _{n \rightarrow \infty} n e_{n}(u) \geq c \cdot\|w\|_{r}
$$

where $1 / r:=1 / p+1 / \alpha$. If we combine (5.13) with Theorem 4.5 we obtain the following.

Proposition 5.3 Suppose $1 \leq p \leq 2$ and $1 \leq \alpha<2$. Let $w$ be a weight function satisfying (5.11). Then

$$
\liminf _{\varepsilon \rightarrow 0} \varepsilon^{\alpha} \cdot\left[-\log \mathbb{P}\left(\int_{0}^{\infty}\left|w(t) Z_{\alpha}(t)\right|^{p} d t<\varepsilon^{p}\right)\right] \geq c \cdot\left(\int_{0}^{\infty} w(t)^{\alpha p /(\alpha+p)} d t\right)^{1+\alpha / p}
$$

for some universal $c>0$. 
This result plays an important role in our further investigation in $\mathrm{Li}$ and Linde ${ }^{[8]}$ on the exact small ball constants for stable processes under weighted $L_{p}$-norm.

Example 3: For $1<\alpha<2$ and $1 / \alpha<H<1$ define the kernel $K$ on $[0,1] \times(-\infty, 1]$ by

$$
K(t, s):=(t-s)_{+}^{H-1 / \alpha}-(-s)_{+}^{H-1 / \alpha}
$$

The S $\alpha$ S-process $X^{H}=\left(X^{H}(t)\right)_{0 \leq t \leq 1}$ generated by this $K$ is usually called linear fractional $\alpha$-stable motion (cf. Samorodnitsky and Taqqu ${ }^{[17]}$ for more information). Let $u: L_{q}[0,1] \rightarrow$ $L_{\alpha}(-\infty, 1]$ be the integral operator with kernel $K$ from above. Then $u$ may be written as

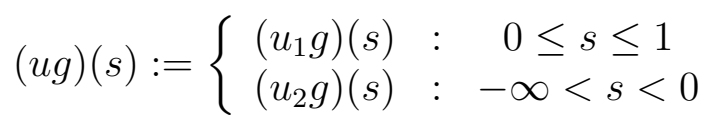

where $u_{1}: L_{q}[0,1] \rightarrow L_{\alpha}[0,1]$ is given by

$$
\left(u_{1} g\right)(s):=\int_{s}^{1}(t-s)^{H-1 / \alpha} g(t) d t, \quad 0 \leq s \leq 1,
$$

while $u_{2}: L_{q}[0,1] \rightarrow L_{\alpha}(-\infty, 0)$ acts as

$$
\left(u_{2} g\right)(s):=\int_{0}^{1}\left[(t-s)^{H-1 / \alpha}-(-s)^{H-1 / \alpha}\right] g(t) d t, \quad-\infty<s<0 .
$$

Of course, for all $g \in L_{q}[0,1]$,

$$
\|u g\|_{\alpha}^{\alpha}=\left\|u_{1} g\right\|_{\alpha}^{\alpha}+\left\|u_{2} g\right\|_{\alpha}^{\alpha} \geq\left\|u_{1} g\right\|_{\alpha}^{\alpha}
$$

and hence (cf. Lifshits and Linde ${ }^{[9]}$ ) we get

$$
e_{n}(u) \geq e_{n}\left(u_{1}\right), \quad n \in \mathbb{N}
$$

On the other hand, by a change of variables $u_{1}$ may be isometrically transformed into $v: L_{q}[0,1] \rightarrow L_{\alpha}[0,1]$ with

$$
(v f)(t):=\int_{0}^{t}(t-s)^{H-1 / \alpha} f(s) d s, \quad 0 \leq s \leq 1
$$

thus $e_{n}(v)=e_{n}\left(u_{1}\right)$. But it is well-known (cf. Edmunds and Triebel ${ }^{[4]}$ or Lifshits and Linde $\left.{ }^{[9]}\right)$ that

$$
e_{n}(v) \approx n^{-(H-1 / \alpha+1)},
$$

and hence by (5.14) we finally obtain

$$
\liminf _{n \rightarrow \infty} n^{H-1 / \alpha+1} e_{n}(u)>0 .
$$

An application of Theorem 4.5 (or Proposition 5.2) to (5.15) implies now the following. 
Proposition 5.4 Let $X^{H}=\left(X^{H}(t)\right)_{t \in[0,1]}$ be a linear fractional $\alpha$-stable motion of order $\alpha \in(1,2)$ and suppose $1 / \alpha<H<1$. Then for any $p \in[1, \infty]$ it follows that

$$
-\log \mathbb{P}\left(\left\|X^{H}\right\|_{L_{p}[0,1]}<\varepsilon\right) \geq c \cdot \varepsilon^{-1 / H} .
$$

For $p=\infty$ this was proved in Samorodnitsky ${ }^{[16]}$ by different methods. It is an open question whether or not $\varepsilon^{-1 / H}$ is the correct order.

Example 4: Our last example deals with an $\mathrm{S} \alpha \mathrm{S}$-process indexed by $[0,1]^{d}$ for some $d \geq 1$. If $u$ from $L_{q}[0,1]^{d}$ to $L_{\alpha}[0,1]^{d}$ is defined by

$$
(u g)(s):=\int_{s_{1}}^{1} \cdots \int_{s_{d}}^{1} g(t) d t_{d} \cdots d t_{1}, \quad s=\left(s_{1}, \ldots, s_{d}\right),
$$

the generated S $\alpha \mathrm{S}$-process $Z_{\alpha}^{d}$ will be called ( $d$-dimensional) $\alpha$-Levy sheet. Note that for $\alpha=2$ we obtain the ordinary $d$-dimensional Brownian sheet. In view of Proposition 11.3.2 in Samorodnitsky and Taqqu ${ }^{[17]}$, for any $p \in[1, \infty)$

$$
\mathbb{P}\left(\int_{[0,1]^{d}}\left|Z_{\alpha}^{d}(t)\right|^{p} d t<\infty\right)=1
$$

An easy transformation gives $e_{n}(u)=e_{n}(v)$ where $v$ from $L_{q}[0,1]^{d}$ to $L_{\alpha}[0,1]^{d}$ is defined by

$$
(v f)(t):=\int_{0}^{t_{1}} \cdots \int_{0}^{t_{d}} f(s) d s_{d} \cdots d s_{1}, \quad t=\left(t_{1}, \ldots, t_{d}\right) .
$$

It is known (cf. Temlyakov ${ }^{[20]}$ ) that for $\alpha \geq 1$

$$
e_{n}(v) \approx n^{-1}(\log n)^{d-1}
$$

and hence Proposition 5.2 applies with $\theta=1$ and $\beta=d-1$ and this leads to

$$
-\log \mathbb{P}\left(\left\|Z_{\alpha}^{d}\right\|_{L_{p}[0,1]^{d}}<\varepsilon\right) \geq c \cdot \varepsilon^{-\alpha} \log (1 / \varepsilon)^{\alpha(d-1)}
$$

for all $p \in[1, \infty]$. It is a challenge to find the correct rate in (5.16).

\section{References}

[1] Bourbaki, N. Integration, Ch. IX Hermann, Paris 1969

[2] Bourgain, J., Pajor, A., Szarek, S. and Tomczak-Jaegermann, N. On the duality problem for entropy numbers of operators. Geometric Aspects of Functional Analysis, Lecture Notes in Math. 1376 (1989), 50-63. 
[3] Chen, X., Kuelbs, J. and Li, W. V. A functional LIL for symmetric stable processes. Ann. Probab. 28 (2000), 258-276.

[4] Edmunds, D. E. and Triebel, H. Function Spaces, Entropy Numbers and Differential Operators. Cambridge Univ. Press. Cambridge 1996.

[5] Kuelbs, J. and Li, W. V. Metric entropy and the small ball problem for Gaussian measures. J. Funct. Anal. 116 (1993), 133-157.

[6] Li, W.V. and Linde, W. Approximation, metric entropy and small ball estimates for Gaussian measures. Ann. Probab. 27 (1999), 1556-1578.

[7] Li, W.V. and Linde, W. Recent developments on small ball probabilities for stable processes. preprint.

[8] Li, W.V. and Linde, W. Small ball probabilities for stable processes under weighted $L_{p}$-norm. preprint.

[9] Lifshits, M. A. and Linde, W. Approximation and entropy numbers of Volterra operators with application to Brownian motion. Memoirs AMS 745 (2002), 1-87.

[10] Linde, W. Probability in Banach spaces - Stable and infinitely divisible distributions. John Wiley \& Sons 1986.

[11] Marcus, M. B. and Pisier, G. Characterizations of almost surely continuous $p$ stable random Fourier series and strongly stationary processes. Acta Math. 152 (1984), $245-301$.

[12] Milman, V. D. and Szarek, S. J. A geometric lemma and duality of entropy numbers. Lect. Notes Math. 1745 (2000), 191-222.

[13] Mogulski, A. A. Small deviations in space of trajectories. Theor. Probab. Appl. 19 (1974), 726-736.

[14] Pajor, A. and Tomczak-Jaegermann, N. Volume ratio and other $s$-numbers of operators related to local properties of Banach spaces. J. Funct. Anal. 87 (1989), 273293.

[15] Ryznar, M. Asymptotic behaviour of stable seminorms near the origin. Ann. Probab. 14 (1986), 287-298.

[16] Samorodnitsky, G. Lower tails of self-similar stable processes. Bernoulli 4 (1998), $127-142$. 
[17] Samorodnitsky, G. and Taqqu, M. S. Stable non-Gaussian random processes. Chapman \& Hall 1994.

[18] Sztencel, R. On the lower tail of stable seminorms. Bull. Polon. Acad. Sci. Math. 32 (1984), 715-719.

[19] Sudakov, V. N. Gaussian measures, Cauchy measures and $\epsilon$-entropy. Soviet Math. Dokl. 10 (1969), 310-313.

[20] Temlyakov, V. N. Approximation of functions with bounded mixed derivative. Trudy Math. Inst. Steklov 178 (1986).

[21] Tortrat, A. Lois $\varepsilon(\lambda)$ dans les espaces vectoriels et lois stables. Z. Wahr. verw. Geb. 37 (1976), 175-182.

[22] Vakhania, N. N., Tarieladze, V. I. and Chobanjan, S. A. Probability distributions in Banach spaces. Nauka Moscow 1985. 